\title{
Second year undergraduate medical student's perceptions, their feedback regarding teaching methodologies and task based learning of pharmacology at Gulbarga Institute of Medical Sciences, Kalaburagi
}

\author{
Harish. G. Bagewadi ${ }^{1}$, Savithri Desai ${ }^{2, *}$, Patil Banderao V ${ }^{3}$, Syed Hasan Zahid ${ }^{4}$ \\ ${ }^{\mathbf{1}}$ Assistant Professor, ${ }^{\mathbf{2}, \mathbf{4}}$ Tutor, ${ }^{3}$ Professor \& HOD, Dept. of Pharmacology, Gulbarga Institute of Medical Sciences, Kalaburagi. \\ Karnataka
}

*Corresponding Author:

Email: savithridesai@gmail.com

\begin{abstract}
Introduction: Pharmacology, like any other branch of medicine, is progressing by leaps and bounds. Consequently, reforms in undergraduate teaching are the need of the hour.

Objectives: To determine the medical students' perception and receive feedback on teaching and learning of pharmacology in our institution.

Material and Methods: This was a cross-sectional study based on the questionnaire. A pre-validated, predesigned questionnaire containing 11 points was administered to fifth term medical students. Second year students were enrolled for the study at their end of $5^{\text {th }}$ term before university exams.

Results: Majority $(80 \%)$ of the students opined that integration of pharmacology knowledge with bed side clinics and analytical way of learning by Problem Based Learning (PBL) enhances their knowledge and skills.

Most $72 \%$ of the students agreed that the best teaching method for learning pharmacology was didactic lectures and group discussions. $96 \%$ of the students agreed that the ideal teaching/learning media for pharmacology was the combination of LCD projector and blackboard. Autonomic Nervous System was the difficult to understand by $(64 \%)$ of the students. $78 \%$ of the students felt that their participation in student seminars has increased our morale, confidence and subject knowledge. $92 \%$ of the students said that prescription writing exercise helped us to know the importance of correct prescription writing.

Conclusion: The present study helped us to elicit student preferences regarding teaching and evaluation methods in pharmacology. Implementation of the same would be helpful for better outcome of students.
\end{abstract}

Keywords: Pharmacology, Teaching, Learning, Opinions, Perceptions, Seminars.

\section{Introduction}

Pharmacology as a discipline is undergoing continuous development and is becoming an important component of all areas of medicine. The good foundation in pharmacology will enable students to develop better prescribing skills for the students in their future. The appreciation and learning the principles of pharmacology and its application in clinical situations play vital role in acquiring the prescribing skills among students. ${ }^{1}$

In view of this, changes in undergraduate teaching are the areas to be looked upon. It is generally agreed that reviewing the teaching program at regular intervals and modifications in the methods of disseminating the knowledge about drugs should be regularly reviewed for the betterment of the students learning. Numerous attempts have been undertaken by many institutions to make the teaching of pharmacology more effective. ${ }^{2}$

Many attempts are tried to make teaching and learning of pharmacology more beneficial all over India. ${ }^{3,4}$ In the present days, newer and innovative teaching \& learning methods like computer assisted learning, usage of audio-visual aids, alternatives to animal experimentation, participation in quiz and seminar activities are playing pivotal role in different task of teaching and learning activities of pharmacology. ${ }^{5,6}$ The best yard stick to assess the effectiveness of different teaching methods is by students feedback and their opinions. Hence, the present study was undertaken to assess the medical students' perception about pharmacology as a subject, its effectiveness in future practice, the teaching and learning methodologies in present times of learning and preparing for examinations at the department of pharmacology, Gulbarga institute of medical sciences, Kalaburagi, Karnataka, India.

Pharmacology, like any other branch of medicine, is progressing comprehensively. It is generally perceived that monitoring the teaching program at regular times and the necessary changes required in disseminating the knowledge about drugs is at most important parameter. Students' feedback would serve as the best tool to assess the effectiveness of teaching and learning methodologies. ${ }^{7}$ Gathering information about people's knowledge, perceptions, attitudes serve as the best entity to look for other modifications that can be done in learning process. ${ }^{8,9}$ Howiit $\mathrm{D}$ and Cramer $\mathrm{D}^{10}$ were of the opinion that questionnaires should be tested for their validity, reliability, standardization and comprehensiveness. The questionnaire is said to be standardized when it is written and distributed to all the subjects and they are asked to give their responses for the relevant same questions in an similar format and the responses are mapped in a uniform manner. ${ }^{11}$ In our 
present study, The questionnaire was designed after the minor modifications of the questionnaires gathered from previous similar studies. ${ }^{12}$ The questionnaire were analyzed and validated by experienced faculties. The study protocol was examined and validated by conducting pilot study for its readability, reliability and comprehensiveness. Knowing the perceptions and their attitude held by the medical students regarding pharmacology and its role in both research and clinical practice will definitely enhance the improvement in teaching and learning methodologies on this subject and relevant modifications into the curricula can be adopted where ever necessary.

In view of this, the present study was conducted to determine the perception and feedback of teaching/learning Pharmacology using a pre-validated questionnaire among $2^{\text {nd }}$ M.B.B.S. (fifth term) students by department of pharmacology at Gulbarga institute of medical sciences, Kalaburagi.

\section{Material and Methods}

The present study was carried out at the department of pharmacology at Gulbarga institute of medical sciences, Kalaburagi. This was a questionnaire based study assessing the attitude, perceptions. Second year medical students were enrolled for the study at their end of $5^{\text {th }}$ term and before the university exams were conducted. Prior permission was obtained from the Institutional Ethics Committee. A questionnaire containing 11 questions were distributed to each student and they were asked to give their response to the best of their knowledge. The questionnaire was based on previous studies undertaken on the evaluation of perception and feedback of teaching/learning in pharmacology and it was suitably modified for our fifth term medical students. The student's identity was not revealed and the generated data was assessed with utmost confidentiality to avoid the bias. The completed questionnaire was collected and data was analyzed. Totally 115 students were participated in the study. So, we have analyzed the responses of 115 participants.

\section{Statistical Analyses}

Data was analyzed and presented as counts and percentages.

\section{Results}

In our study, $84 \%$ of the students (table 1) were of the opinion that Pharmacology lectures were interesting, stimulating and they were more helpful in acquiring the knowledge. Integration of pharmacology knowledge with bed side clinics and discussing the pharmacotherapy and Problem Based Learning (PBL), their rationality of drug usage was more helpful for majority $80 \%$ of the students. $65 \%$ of the students wished to learn recent advances to be included in the curriculum. Majority $63 \%$ of the students were of the opinion that for assessment of their academic performance, Multiple Choice Questions (MCQs) to be included.

From the table 2, it is evident that the combination of LCD projector and blackboard was the best teaching/learning media for pharmacology was opined by the majority (96\%) of students. About $72 \%$ students felt that the ideal teaching method for learning pharmacology was didactic lectures followed by demonstrations (70\%). Among all the systemic pharmacology topics, (64\%) of the students said that the most difficult system to follow and understand was Autonomic Nervous System followed by endocrine system $(38 \%)$.

From table 3 it is observed that $88 \%$ of the students were of the opinion that discussions during pharmacology practical hours regarding different drugs and their dosage forms helped them to gain more knowledge and to know their therapeutic indications.86\% of the students agreed that fixed dose combination exercise made us aware about the rational combinations with their advantages. It was seen that $92 \%$ of the students were of the opinion that Prescription writing exercise helped us to know the importance of correct prescription writing.

\section{Discussion}

The primary objective of teaching pharmacology is to enable undergraduate medical students to write correct rational prescriptions and help them to take rational therapeutic decisions in their future clinical practice. Pharmacology is also one of the most important basic subject which will give strong foundation to the future budding doctors. Due to plenty of recent advances happening in this subject in terms of drug discovery, clinical trials, biosimilars and its update becomes equally important in the medical curriculum. The best yard stick to assess the teaching learning methodology among medical students is their perceptions and attitude. From this, further development and restructuring of medical education can be inculcated in future. Students' feedback is also important for a medical teacher as it serves him also the necessary changes that can be adopted to excel in his career.

In view of this, the present study was conducted to determine the perception and to get the feedback among $2^{\text {nd }}$ M.B.B.S. (fifth term) medical students regarding of teaching/learning methodologies in pharmacology using a pre-validated questionnaire. In the present study, many interesting things came into picture and students suggested many ways that could be incorporated in conventional pharmacology teaching so as to make the subject more interesting and understandable.

In the present study, majority of $80 \%$ of the students wanted case based learning to be introduced into the curriculum whereas in other study by Jai Krishna, et $\mathrm{al}^{7}$ it was opined by $73 \%$ of the students done at MSDS medical college, Fatehgarh. More than half of the students $-58 \%$ of them in the present study wanted group 
discussions to be introduced which was significantly more than the findings of in a another study done by Jai Krishna, et $\mathrm{al}^{7}$ who reported only $31 \%$. In the current study it was observed that majority of $88 \%$ of the students agreed that learning of pharmacology subject should be more closely integrated with the bed side clinical postings from hospitals which will enable them to apply their knowledge wisely and help them to boost their analytical skills. Our study findings are in good correlation with other different studies conducted in New Delhi where $80.46 \%$ students and $87.50 \%$ were in favor of the bedside teaching of clinical pharmacology. ${ }^{12}$ Based on these findings we feel that students learning should be more directed towards discussion of treatment protocols of different admitted cases to know effective decision making process. In our present study majority of $63 \%$ of the students wished that recent advances to be included in the curriculum because these students were of the opinion that they were uncomfortable in answering long and short essay type questions and would rather prefer MCQs over essay type questions for their assessment.78\% of the students felt that their participation in student seminars has increased their morale, confidence and subject knowledge.

Additional feedback included that mnemonics should be more frequently used for the classification and adverse reactions of the drugs, for better remembrance. Some of the students also opined that pharmacology teaching should be more closely integrated with other subjects like microbiology and pathology for better understanding of the pathophysiology of the disease and the different targets for drugs. Our study limitation is that, our results obtained may not be applicable to all the medical students in bigger setup because these findings are based on a single center study from Karnataka. More multi-centric studies need to be carried out among other medical students to draw more correct, conclusive findings. This shows that students found more interest in those practical classes which are clinically oriented and help them in their future clinical practice. Students want to study recent advances in pharmacology even after the end of second year as it will help them in writing rational prescription. Additional suggestions given were to conduct regular test on each topic, to provide hints, pneumonic and flow charts, entrance oriented questions at the end of each topic and to conduct quiz regularly.

\section{Conclusion}

It is important to know the students opinion on teaching of pharmacology and their learning methods so as to modify accordingly the undergraduate teaching program. Developing the new educational strategies to meet the vital objectives of making pharmacology subject more interesting and practicable is the hour of need I the present scenario. We need to identify priority areas for feedback oriented improvement in the pharmacology. The results of our study can serve as positive feedback to make the teaching program more interesting. Multiple choice questions should be included along with the traditional essay type questions and problem-based learning and evaluation may be considered. As teaching and learning is self-evolving process, a well-designed and systematic prospective research needs to be carried out regularly so that students will get updated every year, ensuring the better outcome of a quality efficient healthcare professional.

\section{Acknowledgement}

The authors would like to thank the fifth term medical students for giving their honest feedback and participating in the study.

Table 1: Students' perceptions regarding teaching methodologies

\begin{tabular}{|c|l|c|c|c|}
\hline S. No. & \multicolumn{1}{|c|}{ Items } & Disagree & Neutral & Agree \\
\hline 1 & $\begin{array}{l}\text { I find Pharmacology lectures interesting and } \\
\text { stimulating }\end{array}$ & $8(7)$ & $10(9)$ & $96(84)$ \\
\hline 2 & $\begin{array}{l}\text { I would like application of Pharmacology knowledge } \\
\text { to be more closely integrated with bed side clinics and } \\
\text { Problem Based Learning (PBL) and the rationality of } \\
\text { drug usage }\end{array}$ & $17(15)$ & $6(5)$ & $92(80)$ \\
\hline 3 & $\begin{array}{l}\text { I wish recent advances be included in } \\
\text { Pharmacology curriculum }\end{array}$ & $29(25)$ & $12(10)$ & $75(65)$ \\
\hline 4 & $\begin{array}{l}\text { I would like assessment to be made with inclusion of } \\
\text { multiple choice questions. }\end{array}$ & $18(16)$ & $25(21)$ & $72(63)$ \\
\hline
\end{tabular}

Table 2: Students' opinions about teaching-learning methods in pharmacology

\begin{tabular}{|c|l|c|}
\hline S. No. & Item & $\mathbf{N}(\%)$ \\
\hline 1. & The ideal teaching and Learning media for & \\
& learning Pharmacology subject is: & $62(54)$ \\
& a. LCD projector & $48(42)$ \\
& b. Blackboard & $110(96)$ \\
& c. LCD projector + Blackboard & \\
\hline
\end{tabular}




\begin{tabular}{|c|l|c|}
\hline 2. & The ideal teaching method for learning Pharmacology is: & \\
& a. Didactic lecture & $83(72)$ \\
& b. Tutorial & $75(65)$ \\
& c. Group discussion & $67(58)$ \\
& d. Seminar & $69(60)$ \\
& e. Demonstration & $81(70)$ \\
\hline 3. & The most difficult system to understand in Pharmacology & \\
& is: & $74(64)$ \\
& a. Autonomic Nervous System & $28(24)$ \\
& b. General Pharmacology & $32(28)$ \\
& c. Cardiovascular System & $44(38)$ \\
& d. Endocrine System & $41(36)$ \\
& e. Antimicrobials & $23(20)$ \\
& f. Drugs acting on Gastrointestinal System & $37(32)$ \\
& g. Central nervous system & $12(10)$ \\
& h. Others - Specify & \\
\hline
\end{tabular}

Table 3: Perceptions and practices towards pharmacology teaching and task based learning by the students

\begin{tabular}{|c|l|c|c|}
\hline Sl. No. & \multicolumn{1}{|c|}{ Items } & Yes (\%) & NO(\%) \\
\hline 1. & $\begin{array}{l}\text { Discussions during pharmacology practical } \\
\text { hours regarding different drugs and their } \\
\text { dosage forms and bed side clinics } \\
\text { discussions helped us to gain more } \\
\text { knowledge and to know their therapeutic } \\
\text { indications. }\end{array}$ & $14(12)$ \\
\hline 2 & $\begin{array}{l}\text { Fixed dose combination exercise made us } \\
\text { aware about the rational combinations with } \\
\text { their advantages }\end{array}$ & 99(86) & $12(10)$ \\
\hline 3 & $\begin{array}{l}\text { Our participation in student seminars has } \\
\text { increased our morale, confidence and } \\
\text { subject knowledge }\end{array}$ & $90(78)$ & $25(22)$ \\
\hline 4. & $\begin{array}{l}\text { Prescription writing exercise helped us to } \\
\text { know the importance of correct } \\
\text { prescription writing }\end{array}$ & $106(92)$ & $9(08)$ \\
\hline
\end{tabular}

\section{References}

1. Rangachari PK. Basic sciences in an integrated medical curriculum: The case of pharmacology. Adv Health Sci Educ. 1997;2(2):163-71.

2. Garg PV, Rataboli K. Students' opinion on the prevailing teaching methods in pharmacology and changes recommended. Indian J Pharmacol. 2004;36(3):155-8.

3. Dawane JS, Pandit VA, Dhande PP. A Comparative study of Different Teaching Methodologies used for developing understanding of Cardiac Pharmacology in Undergraduate Medical Students. IOSR-J Research Method Education. 2014;4(3):34-8.

4. Amberkar M, Mohan L, Kumari M, Bairy KL. A Survey on Methods Of Undergraduate Pharmacology Teaching. Asian J Pharmaceutical Research Health Care. 2011:3(2):43-9.

5. Hariharan TS. Need for changes in the practical pharmacology curriculum of medical undergraduates. Indian J Pharmacol. 2004;36(3):181.

6. Gitanjali B, Shashindran CH. Curriculum in clinical pharmacology for medical undergraduates of India. Indian J Pharmacol. 2006;38(S2):108-14.

7. Jai K, Abhishek S, Shwetank G, Aakansha G, Priyamvada S, Mirza URB, et al. Students' current perceptions and feedback on teaching and learning Pharmacology from an evolving medical school. IAIM, 2015; 2(7): 99-104.

8. Oppenheim AN. Questionnaire design, interviewing and attitude measurement. London:continuum, 1992.

9. Sapsford R. Survey research. London: Sage, 1999.

10. Howitt D, Cramer D. First steps in research and statistics. London: Routledge, 2000.

11. Hughes I. Changes in the technological methods of teaching and learning in undergraduate pharmacology in UK higher education. BEE-j, 2003; 1: 1.

12. Manjunath SM, Nagesh Raju G, Srinivas TR, Someswara GM. A study on the evaluation of medical students' perception and feedback of teaching-learning of pharmacology in a medical college. IAIM, 2015; 2(9): 102-110. 